\title{
Screening for mutations in the muscle promoter region and for exonic deletions in a series of 115 $\mathrm{DMD}$ and $\mathrm{BMD}$ patients
}

\author{
L Vitiello, M L Mostacciuolo, S Oliviero, F Schiavon, L Nicoletti, C Angelini, \\ G A Danieli
}

\begin{abstract}
Mutations in the muscle promoter region and exonic deletions were screened in a series of 115 unrelated DMD and BMD patients from north-east Italy. No gross mutations of the promoter region were found. In three cases in which dystrophin of normal size was expressed at low levels, the analysis of DNA sequences of the promoter region failed to detect $a b-$ normalities. The majority of deletions in coding sequences, detected by cDNA probes, occur in the deletion hot spot identified by the probe $\mathbf{P 2 0}$. Intrafamilial variability in the severity of the disease is reported and discussed.
\end{abstract}

Several studies have reported that the great majority of cases affected by Duchenne or Becker muscular dystrophy are the result of deletions in the dystrophin gene, mapping in $\mathrm{Xp} 21$. Most deletions occur in two regions, one of which was first identified by pERT probes $^{1}$ and the second by the probe P20. ${ }^{2}$

No correlation was found between the extent or the position of the deletion and the clinical phenotype, whereas strong correlation exists with the status of the reading frame in the mRNA resulting from deletion. ${ }^{3}$

Immunohistochemistry and immunoblotting showed that $85 \%$ of DMD/BMD patients with unequivocal labelling on blots had a protein of abnormal size, while $15 \%$ had a dystrophin of normal size but of reduced amount, ${ }^{45}$ suggesting the possibility of regulatory mutations. Studies on the regulation and expression of the dystrophin gene led to identification of different promoters, one of which is specific for muscle. ${ }^{6-9}$

The present study deals with the molecular analysis of dystrophin gene mutations in a series of 115 unrelated Italian DMD/BMD patients, with special reference to deletions in the muscle promoter region or in coding sequences or both.

\section{Materials and methods}

Blood samples from unrelated patients with DMD or BMD were mostly referred to the laboratory by the Centre for Neuromuscular Disorders in the Neurology Department, University of Padua, where the patients were diagnosed. The clinical diagnosis was established by muscle histology and clinical assessment. DMD cases were usually differentiated from $\mathrm{BMD}$ by the age at which the patients became chairbound, as suggested by Emery, ${ }^{10}$ or, in younger patients without a family history of the disease, by the results of dystrophin testing. Immunohistochemistry was performed in the laboratory of the same Neurology Department, while immunoblots were done in the laboratory of Dr E Hoffmann, School of Medicine, University of Pittsburgh. Subjects whose muscle specimens proved to be dystrophin negative (less than 3\% compared with normal subjects) were considered to be affected by DMD.

DNA was extracted ${ }^{11}$ from peripheral blood samples obtained in evacuated test tubes coated with K-EDTA (Vacuette, Greiner) and digested with HindIII (Amersham) according to the manufacturer's recommended conditions. Samples were run in 0.9 agarose (Seakem) by reverse field electrophoresis (Hoefer Pulse Controller PC750) in order to improve the resolution of the fragment separation. Agarose gels were blotted onto Hybond-N + (Amersham) membranes. cDNA probes, kindly supplied by Dr L Kunkel, were labelled by random priming (Random priming kit, Promega) with ${ }^{32} \mathrm{P}$-dATP (Amersham). Filters were hybridised at $68^{\circ} \mathrm{C}$ in $7 \% \mathrm{SDS}$, $0.5 \mathrm{~mol} / 1$ disodium phosphate, $1 \mathrm{mmol} / 1$ EDTA, with the addition of $400 \mu \mathrm{g} / \mathrm{ml}$ sonicated and denatured salmon sperm DNA.

After hybridisation, filters were washed four times (20 minutes each) at $68^{\circ} \mathrm{C}$ in $1 \%$ SDS, $50 \mathrm{mmol} / 1$ disodium phosphate, $1 \mathrm{mmol} / 1$ EDTA. Films (Kodak, X-OMAT S) were exposed with intensifying screens for one to six days at $-80^{\circ} \mathrm{C}$.

For reprobing, filters were usually stripped twice in $0.1 \times \mathrm{SSC}, 0.5 \% \mathrm{SDS}$ at $95^{\circ} \mathrm{C}$ for 15 minutes and once for five minutes in $0.1 \times$ SSC at $95^{\circ} \mathrm{C}$.

Primers flanking the sequence of the muscular promoter (Fwd CCCAAGCTTGCAAGTACTATGTCCACTGTGC; Rev CCCGTCGACCACAAACTAAACGTTATGCCACAG) were designed directly from the sequence reported by Klamut $e t$ al $^{12}$ (underlined sequences, containing HindIII and SalI sites respectively, were added for cloning). The sequence between these two primers corresponds to the segment between -217 and +330 and includes the $150 \mathrm{bp}$ segment recognised to be essential for dystrophin gene expression in the muscle cells. ${ }^{12}$

Polymerase chain reaction was performed on a MJResearch Programmable Thermal Controller, using $12.5 \mathrm{pmol} / 1$ of each primer,
Received 3

Revised version accepte 6 June 1991 . 
2.5 units of $T a q$ polymerase (Cetus Co), and $125 \mathrm{ng}$ genomic DNA per $25 \mu \mathrm{l}$ reaction.

Samples were incubated for one minute at $95^{\circ} \mathrm{C}$, followed by one minute at $65^{\circ} \mathrm{C}$ for annealing, and one minute at $72^{\circ} \mathrm{C}$ for elongation. The amplification cycle was repeated 35 times and the reaction ended with five minutes of elongation at $72^{\circ} \mathrm{C}$. Amplification products were run on $1.5 \%$ agarose or $5 \%$ acrylamide gel and subsequently stained by ethidium bromide solution.

Muscle promoters from controls and from patients affected with BMD, amplified by PCR and purified on low melt agarose, were individually cloned in pUC19 using the restriction sites added to the primer sequence.

Plasmid DNA was directly sequenced on both strands using Sequenase $2 \cdot 0$ (USB) with two nested primers (Fwd CACTGTGCTATTCTGGTTTGG; Rev CTTTGTACTTACAACAGTCCTC).

\section{Results}

Deletion screening in $72 \mathrm{DMD}$ and $43 \mathrm{BMD}$ cases was performed by cDNA probes $1-2 \mathrm{a}$, $2 b-3,4-5 a, 5 b-6,7,8$, and 9-10. A representative Southern blot is shown in fig 1 . Deletions were detected in $56.5 \%$ of the DMD/BMD patients. The percentage of deletions was $51 \%$ among DMD patients and $66 \%$ among BMD patients.

Deletion sizes ranged between a minimum of one exon (one DMD and three BMD cases) and a maximum of 43 exons. In one case (DMD) the entire gene sequence was deleted.

The extension and location of the deletions is shown in fig 2. Most deletions occur in the sequences identified by cDNAs 7 and 8, span-

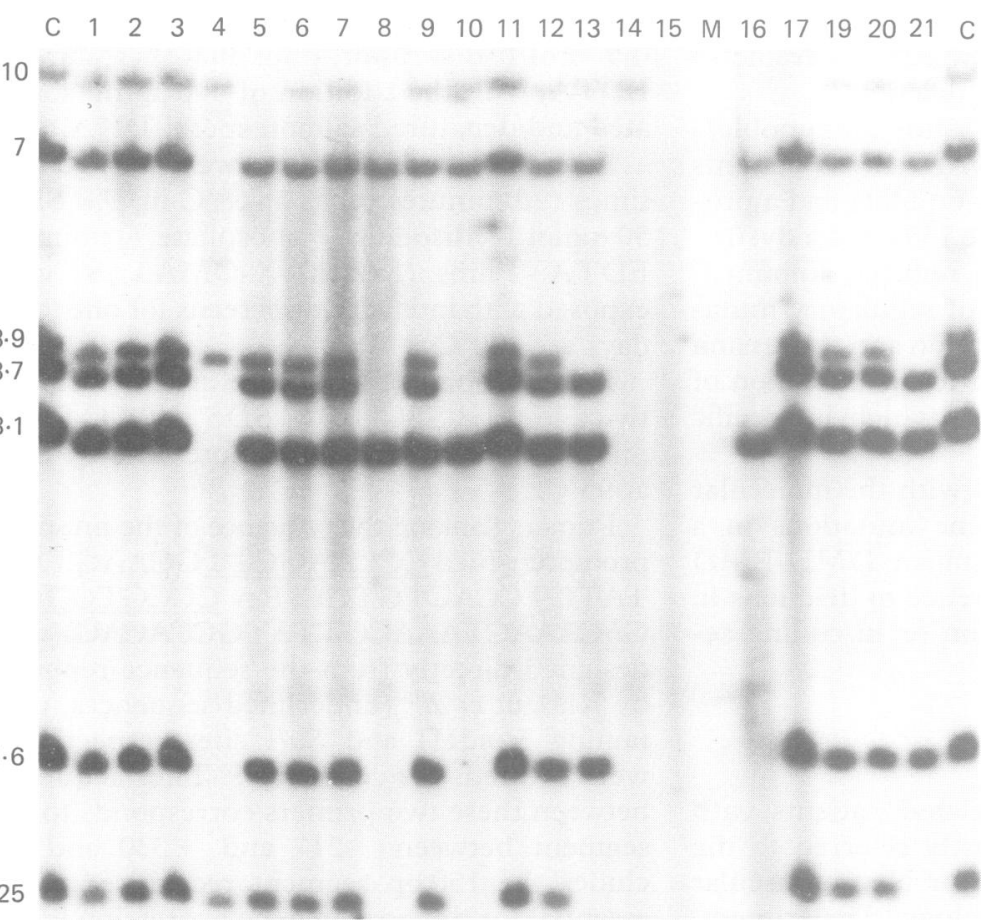

Figure 1 HindIII digested DNA samples from DMD and $B M D(3,5,13,17,21)$ patients probed with cDNA 8. Lanes marked $C$ correspond to the DNA of healthy males. Track $M$ is the DNA molecular weight marker (lambda DNA digested with EcoRI and HindIII). A complete deletion in lane 15 and partial deletions in lanes 4, $5,8,10,13,14,16,17$, and 21 are shown. ning exon 44 to exon 52 . While the $5^{\prime}$ deletion breakpoint in this region is variable in DMD cases, in $70 \%$ of $\mathrm{BMD}$ cases it lies between exons 44 and 45 , confirming previous observations obtained by hybridisation with the P20 intragenic probe. ${ }^{13-15}$ In 57 cases (28 DMD and 29 BMD) the status of the reading frame resulting from deletion was established. Among DMD patients, $96 \%$ (27 out of 28 ) were out of frame and among BMD patients $96 \%$ ( 28 out of 29 ) were in frame.

Clinical variability among affected subjects in the same family was observed in several instances, as shown in fig 3. All 35 DMD patients whose DNA did not show deletions in the coding sequences and three BMD cases with reduced amounts of normal dystrophin were tested for deletions in the muscle promoter region by PCR amplification and subsequent gel electrophoresis (fig 4).

None of these 38 patients showed deletions in the segment that includes the muscle promoter. As expected, no promoter deletions were found among patients showing exonic deletions or showing a dystrophin altered in size.

The muscle promoter regions of the three BMD patients showing reduced levels of normal dystrophin (less than $50 \%$ of normal) were cloned in pUC19 and sequenced. Four independent clones were analysed for each patient and for two unrelated healthy male controls. The comparison of sequences showed minor variations in one control and in one BMD patient (deletion of a $T$ in a series of seven in one, and in a series of eight in the other), but no significant differences were present (data not shown)

\section{Discussion}

The absence of gross mutations in the region of the muscle promoter is not surprising, because of the small size of the sequence involved and the presumed selective pressure for the conservation of consensus sequences. A deletion of the promoter region has only been described once so far. ${ }^{9}$ The rarity of this kind of mutation is confirmed by the present study, where no promoter deletions were found in a series of 115 unrelated patients.

None of the three BMD cases with reduced dystrophin of normal size showed mutations in the sequence including the muscle promoter, but the reduction of dystrophin molecules might be the result of point mutations in the coding sequence. Such mutations could make the protein more prone to rapid degradation. Alternatively, the observed reduction of dystrophin could be explained by mutation in still undefined additional regulation sites.

The large majority $(86 \%)$ of $5^{\prime}$ deletion breakpoints found in the present study map between the exons identified by cDNA probes 7 and 8. The occurrence of deletion breakpoints in two hotspots has been pointed out in several reports, ${ }^{316-18}$ but here only $11.5 \%$ of DMD deletions and $10 \%$ of BMD deletions did not include exons 40 to 55 . These percentages are much lower than previously reported. The difference can hardly be attributed to the 


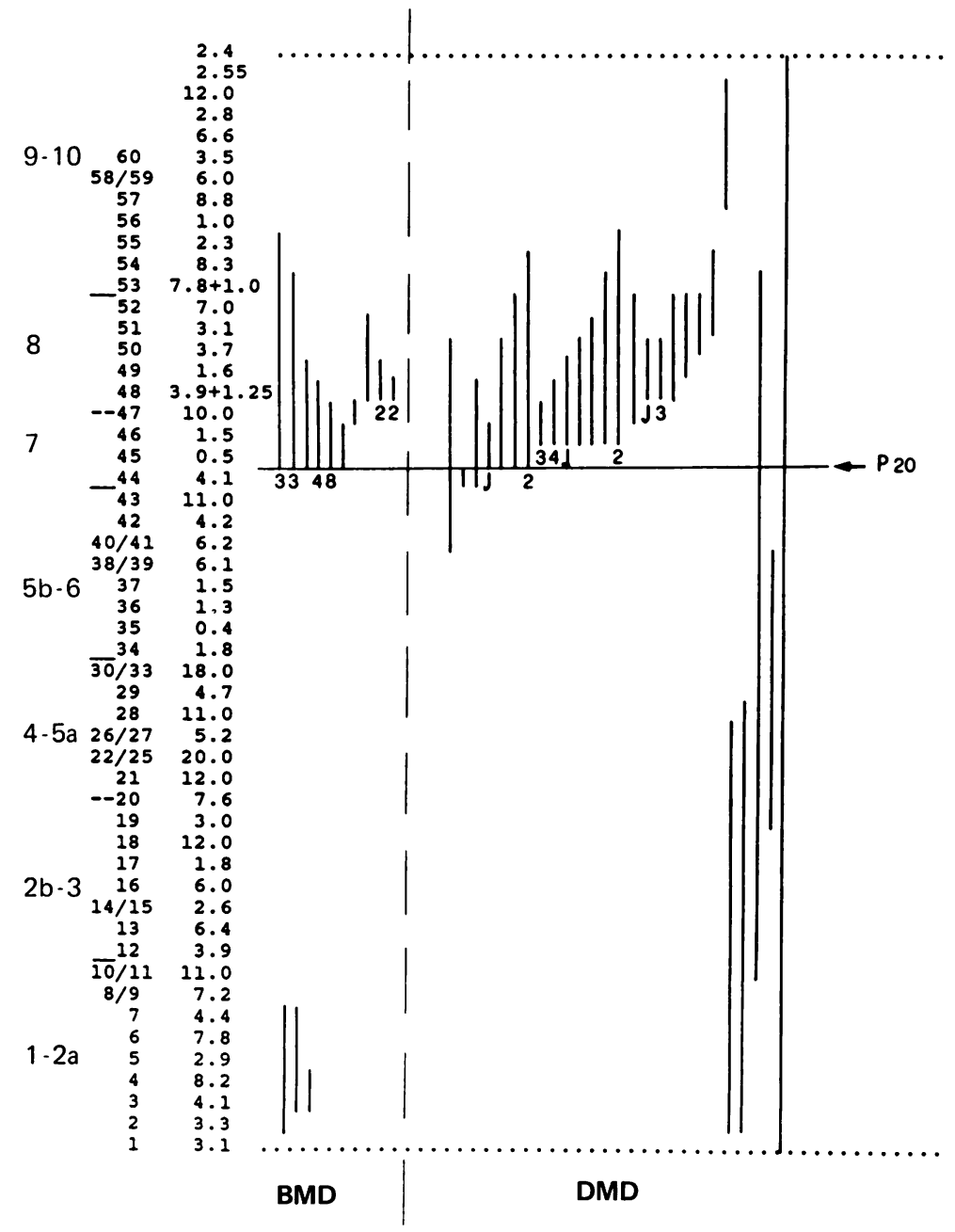

Figure 2 Summary of 65 deletions found among 115 unrelated patients affected with $D M D$ or BMD. From left to right: $c D N A$ probes used in the study; exon numbers; corresponding HindIII fragments (in $k b$ ) detected by $c D N A$ probes; extent of deletions among BMD patients; extent of deletions among DMD patients. Numbers below the bars indicate the number of patients carrying the same deletion. $\mathcal{F}$ indicates the presence of a junction fragment. The horizontal line shows the location of the intragenic probe P20 (see text for explanation). size of the sample. Perhaps the homogeneity of the location of the $5^{\prime}$ deletion breakpoints results from the geographical homogeneity of the sample. Persons who share common ancestors are likely also to share homologous noncoding sequences of DNA. Since deletions occur preferentially in specific introns, a differential deletion pattern could actually exist in different populations. In the present study, $50 \%$ of DMD and $73 \%$ of BMD cases were from one geographical area of 66722 square $\mathrm{km}$ (north-east Italy), where there has been little migration and where the internal mobility is still quite low. The relationship between the location of deletions and clinical phenotypes confirmed the frameshift hypothesis, ${ }^{19}$ although in one DMD case (deletion $10 \rightarrow 53$ ) the correct frame was maintained.

A similar in frame deletion $10 \rightarrow 53$ was reported by Koenig $e t a l^{\beta}$ in a DMD patient, whereas a deletion of comparable location and slightly smaller size $(17 \rightarrow 48)$ was reported by Davies et $a l^{20}$ in a very mild Becker case. According to these observations a large part of the rod in the dystrophin molecule seems to be dispensable, but the two ends of the rod seem to be necessary for maintaining partial activity of the deleted molecule.

As far as the BMD cases are concerned the only out of frame deletion encompasses exons $3 \rightarrow 7$. Similar deletions were reported by Malhotra $e t a l^{21}$ and by Koenig et al. ${ }^{3}$ The clinically benign course in these cases was attributed to alternative splicing, as recently confirmed by sequencing. ${ }^{22}$

One relatively large deletion (exons $45 \rightarrow 55$ ) was found in the present investigation in a man, aged 31, who suffered only from myalgia after heavy muscular exercise and who has mild hypertrophy of the calves. The same deletion $(45 \rightarrow 55)$ was found in an unrelated BMD patient, aged 64 , who walked with great difficulty, and in another BMD patient, now 25 years old and still walking, who came to
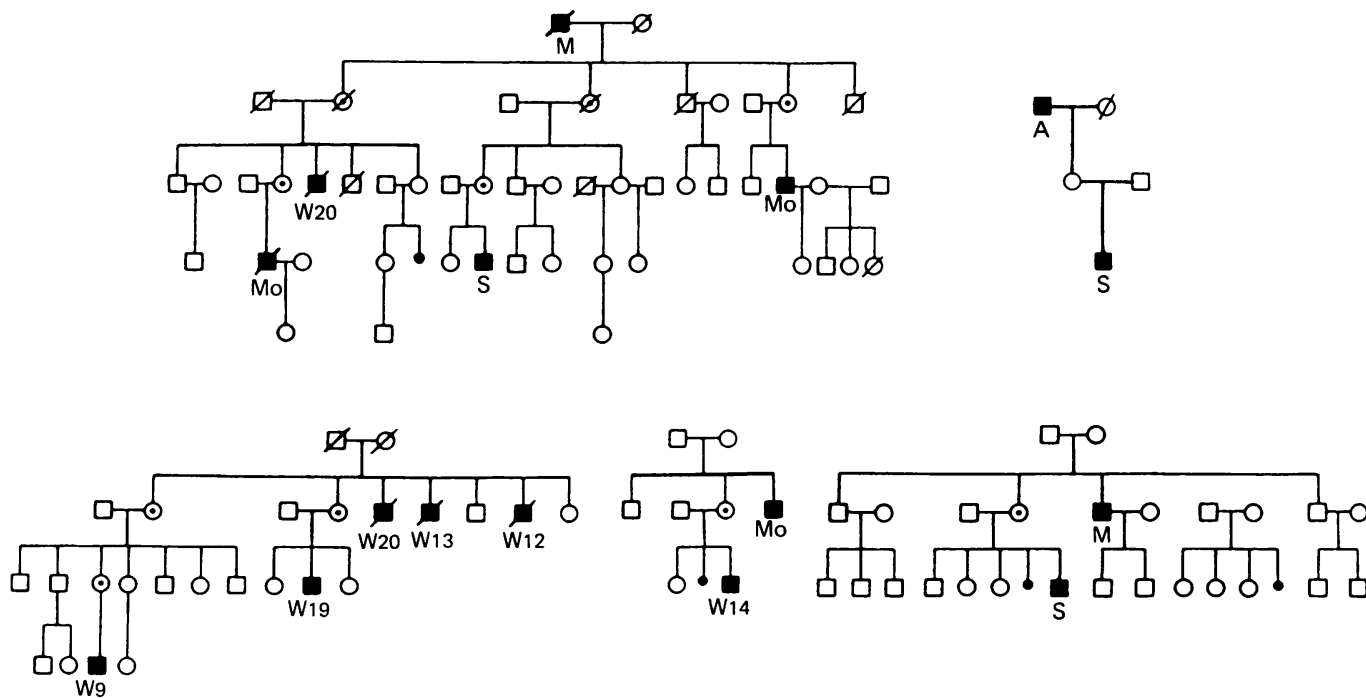

Figure 3 BMD families showing variability in the clinical phenotype. The severity of the disease is indicated as follows: $A$, asymptomatic (raised $C K$ level or calf hypertrophy or both); $M$, mild (same symptoms as above plus clinically detectable weakness and fatigue after physical exercise); Mo, moderate (same symptoms as above plus waddling gait, difficulties in climbing stairs, and clear muscular weakness); $S$, severe (same symptoms as above plus muscular wasting, loss of most muscular strength, and great difficulty in walking); $W$, wheelchair bound. Numbers indicate the age at which the patients lost the ability to walk. 


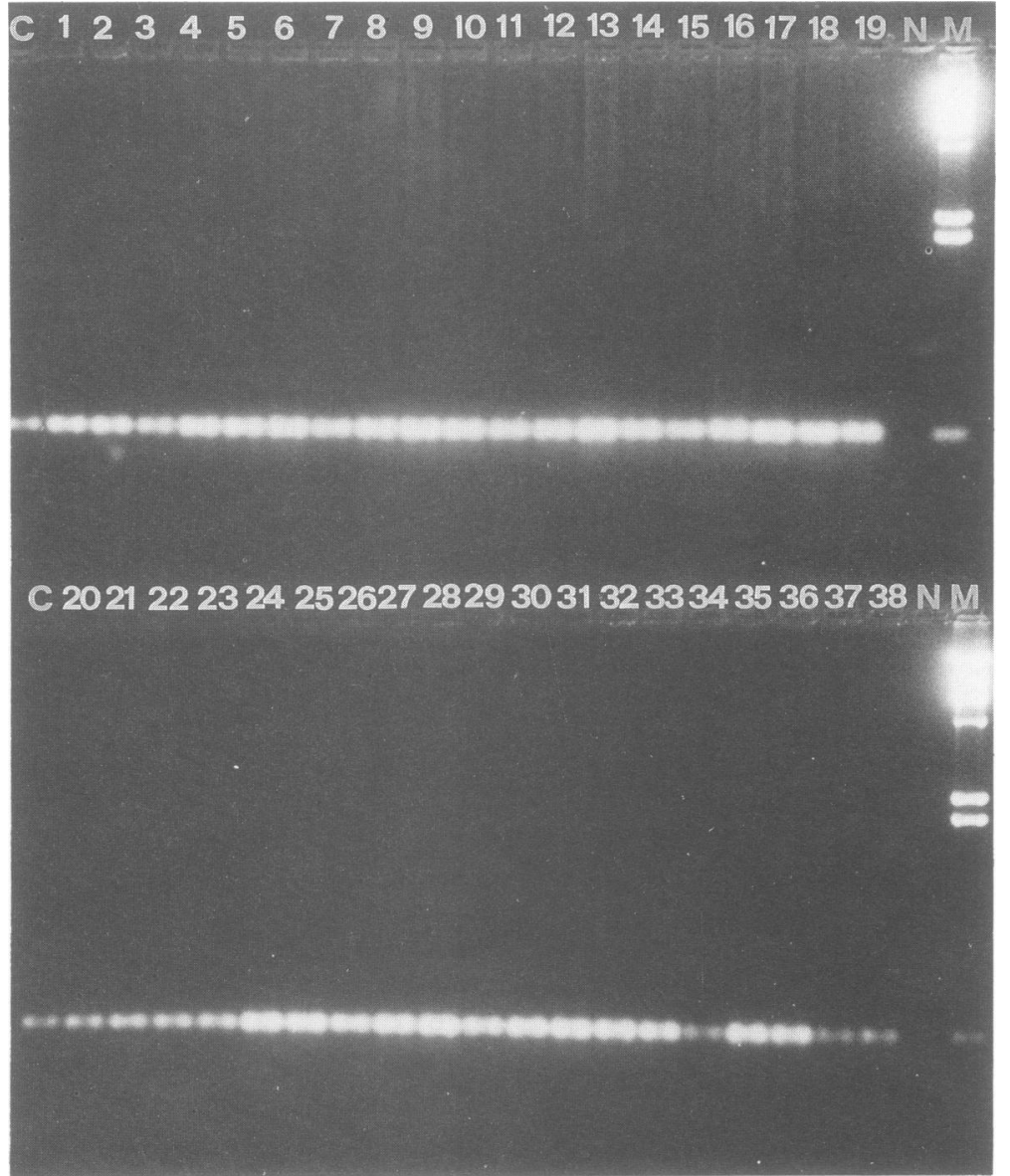

Figure 4 Screening for gross mutations of the muscle promoter region by $P C R$ amplification followed by agarose gel electrophoresis of the resulting $568 \mathrm{bp}$. Samples 4 to 38 are from 35 BMD non-deleted patients. Samples 1, 2, and 3 are from three BMD patients showing reduced amount of normal sized dystrophin. Positive controls (DNA from healthy males) were run in lanes $C$ and negative controls in lanes $N$. The last lanes on the right correspond to the DNA molecular weight marker (lambda DNA, digested with HindIII).

medical attention at the age of 18 because of a very high $\mathrm{CK}$ level.

In the BMD families with recurrence of the disease, large variability in the clinical features of the related patients is observed in different generations (fig 3). It is a general observation that the clinical course is very similar among brothers; therefore the occurrence of cases with different grades of severity in different generations raises the suspicion that additional factors may play a role in the expression of the clinical phenotype. Although the absence or alteration of dystrophin has been identified as the primary cause of DMD and BMD, the subsequent steps of the molecular pathogenesis are still unknown. It is not difficult to accept that genetic variability may exist in molecules involved in key processes like recognition and destruction of damaged muscle fibers, muscular regeneration, or simply in the cellular response to different hormones.

The collaboration of Dr E Hoffman, Department of Molecular Genetics and Biochemistry, School of Medicine, University of Pittsburgh in providing western blot confirmation of dystrophin assessment by immunohistochemistry and the active participation of Drs E Pegoraro and $M$ Fanin in clinical and laboratory assessment of cases are gratefully acknowledged. The work was done with financial support of the Telethon Research Fund, UILDM (Italian Muscular Dystrophy Association), and the Italian Ministry of University and Scientific Research (MURST).

1 Monaco AP, Bertelson CJ, Middlesworth W, et al. Detection of deletions spanning the DMD locus using a tightly linked DMD segment. Nature 1985;316:842-5.

2 Wapenaar MC, Kievits T, Hart KA, et al. A deletion hot spot in the DMD gene. Genomics 1988;2:101-8.

3 Koenig M, Beggs AH, Moyer M, et al. The molecular basis of Duchenne versus Becker muscular dystrophy: correla tions of severity with type of deletions. Am $\mathfrak{\jmath}$ Hum Genet 1989;45:498-506.

4 Hoffmann EP, Kunkel LM, Anglini C, Clarke A, Johnson M, Harris JB. Improved diagnosis of Becker muscular M, Harris JB. Improved diagnosis of Becker muscular
dystrophy by dystrophin testing. Neurology 1989; dystrophy

5 Nicholson LVB, Johnson M, Gardner-Medwin D, Bhattacharya $S$, Harris JB. Heterogeneity of dystrophin expression in patients with Duchenne and Becker muscular dystrophy. Acta Neuropatol 1990;80:238-50.

6 Klamut HJ, Zubrzycka-Gaarn EE, Bulman DE, et al. Myogenic regulation of dystrophin gene expression. $\mathrm{Br}$ Med Bull 1989;45:681-702.

7 Chelly J, Hamard G, Koulakoff A, Kaplan JC, Kahn A Berwald-Netter Y. Dystrophin gene transcribed from different promoters in neuronal and glial cells. Nature 1990;344:64-5.

8 Yaffe D, Makover A, Bar S, Zuk D, Barnea E, Nudel U. Transcripts of the Duchenne muscular dystrophy gene in non-muscular tissues. In: Angelini C, et al, eds. Muscular dystrophy research. From molecular diagnosis towards therapy. Amsterdam: Elsevier, 1991.

9 Boyce FM, Beggs AH, Feener C, Kunkel LM. Dystrophin is transcribed in brain from a distant upstream promoter. Proc Natl Acad Sci USA 1991;88:1276-80.

10 Emery AEH. Duchenne muscular dystrophy. Oxford: Oxford University Press, 1987.

11 Miller SA, Dykes DD, Polesky HF. A simple salting out procedure for extracting DNA from human nucleated procedure for extracting DNA from
cells. Nucleic Acids Res 1988;16:1215.

12 Klamut HJ, Gangopadhyay SB, Worton RG, Ray PN. Molecular and functional analysis of the muscle-specific promoter region of the Duchenne muscular dystrophy promoter region of the Duchenne

13 Gilgenkrantz H, Lambert M, Recan D, et al. Deletions du gene DMD (dystrophine) detectees a l'aide de sondes introniques et exoniques. Proc 3rd Colloque National sur le Maladies Neuromusculaires, Bordeaux, 1988 (abstract).

14 Gilgenkrantz H, Chelly J, Lambert M, Recan D, Barbot JC. Analysis of molecular deletions with CDNA probes in patients with Duchenne and Becker muscular dystrophy Genomics 1989;5:574-80.

15 Vitiello L, Giro E, Mostacciuolo ML, Mueller E, Daniel GA. Deletions in the distal half of the DMD-BMD locus revealed by the intragenic probe P20. Atti Associazione Genetica Italiana 1989; XXXV:387.

16 Den Dunnen JT, Grootscholten PM, Bakker E, et al. Topography of the Duchenne muscular dystrophy gen (DMD): FIGE and cDNA of 194 cases reveals 115 deletions and 13 duplications. Am $\mathcal{f}$ Hum Genet 1989; 45:835-47.

17 Gillard EF, Chamberlain JS, Murphy EG, et al. Molecula and phenotypic analysis of patients with deletions within the deletion-rich region of the Duchenne muscular dysthe deletion-rich region of the Duchenne muscular dys-

18 Cooke A, Lanyon WG, Wilcox DE, et al. Analysis of Scottish Duchenne and Becker muscular dystrophy families with dystrophin cDNA probes. $f$ Med Genet 1990;27:292-7.

19 Monaco AP, Bertelson CJ, Liechti-Gallati S, Moser H, Kunkel LM. An explanation for the phenotypic difference between patients bearing partial deletions of the DMD locus. Genomics 1988;2:90-5.

20 England SB, Nicholson LVB, Johnson MA, et al. Very mild muscular dystrophy associated with the deletion of $46 \%$ of the dystrophin. Nature 1990;343:180-2.

21 Malhotra SB, Hart KA, Klamut HJ, et al. Frame-shif deletions in patients with Duchenne and Becker muscula dystrophy. Science 1988;242:759-66.

22 Chelly J, Gilgenkrantz $\mathrm{H}$, Lambert $\mathrm{M}$, et al. The dystrophin transcripts in DMD and BMD patients with gen deletions. In: Angelini C, et al, eds. Muscular dystrophy research. From molecular diagnosis towards therapy. Amsterdam: Elsevier, 1991. 